\title{
Relative Fairness and Optimized Throughput for Mobile Ad hoc Networks*
}

\author{
Abderrahim Benslimane, Abderrezak Rachedi and Deepak Diwakar \\ LIA/CERI, University of Avignon, Agroparc \\ BP 1228, 84911 Avignon, France \\ Email: abderrahim.benslimane@univ-avignon.fr
}

\begin{abstract}
Although IEEE 802.11 provides several transmission rates, a suitable rate adaptation taking into account the relative fairness among all competitive stations, according to the underlying channel quality remains a challenge in Mobile Ad hoc Networks (MANETs). The absence of any fixed infrastructure and any centralized control makes the existing solutions for WLANs like CARA (collision-aware rate adaptation) not appropriate for MANETs. In this paper, we propose a new analytical model with a suitable approach to ensure a relative fairness among all competitive nodes of a particular channel. Our model deals with the channel quality while respecting the nodes, based on transmission successes and failures in a mobility context. Finally, each node calculates its own probability to access the channel in a distributed manner. We evaluate the performance of our scheme with others in the context of MANET via extensive and detailed simulations. The performance differentials are analysed using varying network load and transmission range. The simulation results illustrate that our proposed approach ensures a better tradeoff between fairness and throughput.
\end{abstract}

Keywords- IEEE 802.11 MAC, Multihop, Ad hoc network, distributed algorithm, Relative Fairness

\section{INTRODUCTION}

Over the past years, wireless ad hoc networks have awaken critical opinions, that are still growing in the networking research community. IEEE 802.11 implements access methods for sharing the air medium in both cases: wireless LAN (which is centralized) and ad hoc networks (which is decentralized). The Distributed Coordination Function (DCF) is the fundamental MAC technique of the IEEE 802.11 [6]. It is based on Carrier-Sense Multiple Access and Collision Avoidance (CSMA/CA). 802.11 provides various transmission rates. In 802.11a, for instance, some discrete rates are available from $6 \mathrm{mbps}$ to $54 \mathrm{mbps}$ and in the most widely used 802.11 b system/option, four different transmission rates are available, such as $\{1,2,5.5,11\}$ mbps. Although we have various transmission rates available in 802.11 , there is no standard approach defined in 802.11 to select the appropriate rate for a specific condition and to ensure fairness among the competitive nodes. Various rate adaptation schemes are proposed, and take into account the Signal-to-Interference plus Noise Ratio (SINR), the Auto Rate Fall-back, the receiver feedback approach to CollisionAware Rate Adaptation schemes. But they are not suitable for Mobile Ad hoc Networks (MANETs). A node can not adapt itself to its rate without taking into account the other

\footnotetext{
* This work is supported by the ANR "Agence Nationale de la Recherche -France" within the project framework ARA/CLADIS.
}

competitive nodes, unlike in the centralized case. Even though it has appropriate or sufficient information about the other nodes, which are all its competitive nodes, it has no control over them. But this is not the case in Wireless Local Area Network (WLAN). If it does so in MANET, then that may become unfair with other competitive nodes.

IEEE 802.11 standard provides various transmission rates, but it does not specify any algorithm or protocol to efficiently use these rates, in order to make competitive nodes fair . The effectiveness of any rate adaptation scheme depends on how it is incorporating the effect of transmission failures (that may be caused by a channel error or by a collision). Many rate adaptation schemes have been proposed, for example in [2], [5], [1] and [4], but unfortunately none of them properly takes into account this effect in their schemes. In RBAR [5], based on SNR values, the receiver decides the next rate for the sender. But the receiver may not have a correct interpretation of the sender channel and other competitive nodes of the sender. Hence, it does not take into account the fairness among competitive nodes. In Automatic Rate Fall-back (ARF) [1], the sender deduces the channel condition by measuring consecutive successful and failed transmissions and adjusts its rate in accordance with them. But even in this scheme, the sender does not bother about other competitive nodes and adopts its rate without taking them into account. So, it is quite clear that ARF is not appropriate for MANETs to ensure fairness among active nodes, although it is widely used in WLAN. In [4] the authors tried to distinguish channel error and collision. A sender decrements its rate only upon a few consecutive transmission channel errors and increments upon some consecutive transmission successes. In some cases this approach gives a significant improvement to the throughput, compared to the previous schemes. However, this scheme does not take into account other competitive nodes, and in the case of MANET, it does not ensure fairness among competitive nodes.

In this paper, we propose an analytical model to select the rate, and this model takes into account the impact of competitive nodes, in order to create a relative fairness among the competitive nodes without significantly affecting the system throughput. This implies that a tradeoff has to be found between both of them. We propose a new approach called REFOT (Relative Fairness and Optimized Throughput), in order to ensure a balance point between absolute 
fairness and throughput. REFOT has the same principle as CARA (Collision-Aware Rate Adaptation), but it is adapted to MANETs' characteristics. We assess the channel quality according to the transmission failures/successes. Each competitive node belongs to the sender's transmission range. Competitive nodes can have access to the channel under different conditions, that ensure that the channel quality is evaluated by the sender before it selects its rate. Each node calculates the probability to access the channel by taking into account all its competitive nodes. A sender updates this probability each time it wants to send data.

The rest of the paper is organized as follows. In section 2 , we present a few concepts and already existing techniques. Section 3 describes our analytical model. In section 4, we show the simulation setup, analysis and results. Finally, section 5 concludes the paper.

\section{PRELIMINARIES}

In this section, we briefly present IEEE 802.11 in DCF mode, RTS/CST mechanism and some existing rate adaptation techniques based on 802.11.

\section{A. IEEE 802.11 DCF}

IEEE 802.11 Distributed Coordination Function (DCF) mode [6] combines Carrier Sense Multiple Access/Collision Avoidance (CSMA/CA) with a Request to Send/Clear to Send (RTS/CTS) handshake to avoid collisions. It works as follows: when a node wants to transmit a packet, if the node senses the medium idle for a period of time longer than or equal to a Distributed Inter Frame Space (DIFS), the packet transmission may begin with the following slot. Otherwise, the node should backoff for a certain period based on a value randomly selected from $[0, \mathrm{CW}]$, where $\mathrm{CW}$ is the contention window size. The backoff value $\mathrm{cw}$ is initially randomly selected from the range [0,CWmin], where $C W \min =2^{i m i n}-1 . \mathrm{CW}$ is doubled if the transmitted packet fails, e.g., due to collisions or experiencing CRC errors. CW keeps on increasing until it reaches the upper bound $\mathrm{CWmax}$, where $C W \max =2^{i \max }-1$. Each time a packet transmission is successful, $\mathrm{CW}$ is reset to $\mathrm{CWmin}$. Although DCF has a random backoff, it still can't ensure collision-free transmissions, because it might be possible that two or more nodes finish the backoff simultaneously. Beside collisions, transmission failures may also be caused by channel errors. Upon successful contention for the channel, the node requests the channel by sending a RTS to the receiver which, in return, replies with a CTS. The nodes in the vicinity that overhear the RTS or CTS defer their own transmission for a period that is long enough to let the subsequent DATA/ACK exchange happen. When the RTS/CTS handshake is completed, the sender starts the data transmission. The receiver acknowledges the data with an ACK. If no CTS or ACK is received, the sender exponentially backs off, and retransmits the RTS or the DATA. The scheme is called in this paper "Classical RTS/CTS". Since the number of transmission's retries is bounded by ssrc (station short-frame retry counter) and slrc (station long-frame retry counter), a packet is dropped after limited retries.

Usually, the RTS frame is used when the size of pending data is larger than the RTS_threshold value. But using RTS/CTS handshake before every data transmission wastes the time of data transmission, mainly in the case where there is no such hidden terminal problem. Again this scheme does not take into account the channel quality and the effect of the presence of other competitive nodes in MANET and hence it can create unfairness among them.

\section{B. IEEE802.11 ARF}

ARF developed for Lucent Technology' WaveLAN-2 WLAN device [1], has widely implemented a rate adaptation scheme because of its simplicity. According to this scheme, if two consecutive ACKs are not received correctly, then the next retry of data transmission takes place at a lower rate and a timer is started. If a node receives 10 consecutive successful ACKs or if the timer expires, then the next transmission takes place at the next higher rate and the timer is set to zero. Indeed, ARF does not address the reason of transmission failures, i.e., channel errors or frame collisions. A node decreases its rate even though transmission failures caused by collisions which is not appropriate to have a better throughput. At the same time, this scheme does not take into account other competitive nodes and hence in MANETs, it may become unfair among active nodes.

\section{CARA (Collision-Aware Rate Adaptation)}

An adaptive use of RTS/CTS exchange is the core idea of this scheme. Unlike ARF, this scheme tries to distinguish the causes of transmission failures: collisions and channel errors. According to this distinction, a transmitter decrements its rate only on some consecutive channel errors, not on collisions. And hence this scheme outperforms ARF in many cases. This scheme uses probing of RTS/CTS to enable RTS/CTS exchanges. Although it has a better assessment of the channel quality than ARF and Classical RTS/CTS, it is well adapted for WLAN but not for MANET. CARA does not take into account the relative fairness among the competitive nodes that have access to the channel. In this paper, we focus on the relative fairness among the competitive nodes that have access to the channel. We give that balance between absolute fairness and throughput optimization.

\section{REFOT: RELATIVE FAIRNESS AND OPTIMIZED THROUGHPUT}

In this section, we present the model and show how to create a relative fairness among a channel's competitive nodes before adapting the rate. REFOT does not need any centralized coordination; it is adapted to any topology change and it takes into account MANETs' characteristics. Furthermore, REFOT ensures that the adaptation rate takes the other competitive nodes into account, in order to avoid unfair situations between competitive nodes without any degradation of the throughput. 
Like in CARA, each node maintains variables to count consecutive transmission failures $(n)$ and consecutive transmission successes $(m)$. When the value of $n$ reaches the RTS probing threshold $\left(P_{t h}\right)$, the transmitter enables the RTS/CTS exchange before sending data. If $n$ reaches the consecutive failure threshold $\left(N_{t h}\right)$, the transmitter decrements its rate to a lower available rate. But, if $m$ reaches the consecutive success threshold $\left(M_{t h}\right)$, the transmitter increments its rate to the next higher available rate. Moreover, we introduce two variables: the total number of transmissions $(K(t))$ and the total number of transmission failures $(f(t))$ till the current time $t$.

We also consider different transmission rates available in 802.11b $\{1 \mathrm{Mbps}, 2 \mathrm{Mbps}, 5.5 \mathrm{Mbps}, 11 \mathrm{Mbps}\}$. We note this set $R \_d t$ and the current rate $r$. When the value of $n$ reaches $P_{t h}$, RTS/CTS probing takes place and when this value ( $n$ ) reaches $N_{t h}$, a transmitter chooses the lowest transmission rate in the $R \_d t$ set. When the value of $m$ reaches $M_{t h}$, the transmitter chooses the next available higher rate from $R \_d t$ set. Hence, a transmitter knows the transmitting rate $(r)$ at which it is going to transmit the data frame. In REFOT, a node observes its transmission failures and by taking into account the other competitive nodes, it calculates its probability to have access to the channel which is then used to calculate the backoff delay. First, a node $i$ computes the probability of transmission failure $P_{(i, f)}$; this probability is computed according its own experience of transmissions and failures:

$$
P_{(i, f)}=\frac{f_{i}(t)}{K_{i}(t)}
$$

A channel is defined as the radio area shared by the sender's competitive nodes. Logically, it represents the sender's carrier sense $(C S)$. Let us consider a node $\mathrm{i}$ which belongs to the channel $J$. Then, we define a new metric to measure the channel quality. So, a node i computes the probability that the transmission fails within its current channel $J, P_{(i, J)}$. Unlike the probability that the transmission fails, $P_{(i, J)}$ is the vision of node $i$ on the state of its main channel $J$ (i.e., its carrier sense) at the current time. We represent channel $J$ as a set of neighbors in the carrier sense of node $i$ at time $t$. To this aim, each node overhears the packets transmitted in its carrier sense in order to collect the transmission rate, the probability of transmission failure $P_{(l, f)}$ and $P_{(i, J)}$. Then, the probability that the transmission of node $i$ fails within its current channel $J$ is defined as follows:

$$
P_{(i, J)}=\frac{\sum_{l \in J} P_{(l, f)} r_{l}}{\sum_{l \in J} r_{l}}
$$

where $r_{l}$ is the transmission rate of node $l$.

Each node $\mathrm{i}$ informs its neighbors about its current rate value $r_{i}, P_{(i, f)}$ and $P_{(i, J)}$ at each transmission. For example, we can use the Address 4 field ( 6 bytes) in the MAC header since it is not needed in ad-hoc mode [6]. Then each node calculates the probability to access the main channel as follows:

$$
P_{(i \text { Accesses })}=\frac{1-P_{(i, J)}}{\sum_{i \in J}\left(1-P_{(i, J)}\right)}
$$

This probability takes into account the neighbor nodes' behaviour. Each node evaluates its socialization in their society (channel). According to this metric, each node respects each other to access the communication channel. Then this probability is incorporated in order to determine the value of the lowest bound of the transmitter node's backoff window $C W_{\min }^{*}$ as follows:

$C W_{\text {min }}^{*}= \begin{cases}C W_{\min } \cdot\left(1-P_{(\text {iAccess })}\right) & \text { if } P_{(i \text { Access } J)} \neq 1 \\ C W_{\min } & \text { Otherwise }\end{cases}$

When a new node arrives, it has no information about its neighbors. So, the value of its $P_{(i A c c e s s J)}$ is initiated to 1 , in order to prevent the new node from having immediately access to the channel. In that way, the backoff window of all competitive nodes is affected. The channel access of these nodes is dependent on the channel quality which is measured by the probability of transmission failure within the channel of node. This mechanism creates a relative fairness among competitive nodes. It also permits a good rate adaptation before the transmission. We have shown in the simulation's results that without affecting the average system's throughout significantly, we have a much better fairness index in comparison with CARA and classical RTS/CTS schemes, and that proves that our approach creates a relative fairness among competitive nodes, since we know that creating fairness and optimizing the throughput have a tradeoff. In order to understand this tradeoff, let us consider a simple example of a network consisting of 3 nodes with a static topology, in which there are two senders and one receiver. One sender is transmitting with a constant rate of $10 \mathrm{Mbps}$ and another is transmitting with a rate of 1 Mbps, and both are sending data to the same receiver. In the optimal case, the maximum throughput per second can reach $10 M$ bps if only the former gets a chance to transmit data. But if we create an absolute fairness (say let both transmit data for an equal time interval) then the throughput reaches only $5.5 \mathrm{Mbps}$. Our scheme has a balance point between creating a relative fairness and maximizing the throughput.

In order to study the fairness parameter, we use the Fairness Index $(F I)$ as metric. The Fairness Index of a topology is defined as follows:

$$
F I=\frac{\sum_{i=1}^{N} P_{(i \text { Access } J)} r_{i}}{\sum_{i=1}^{N} r_{i}}
$$

where $N$ is the total number of nodes in the mentioned network topology. The probability to access the channel for each node and the transmission rate are important parameters in equation 4 , which permits to calculate the $F I$. In REFOT, we give a new definition of $P_{(i \text { Access } J)}$ like in equation 3 . But for others, like CARA and Classical RTS/CTS schemes, $P_{(i \text { AccessJ })}$ is calculated as follows:

$$
P_{(i \text { Access })}=\frac{1}{\text { Number of neighbouring nodes }}
$$

where the number of neighbor nodes can be estimated by overhearing (we assume that a node can overhear the transmission of the other nodes who fall into its transmission 
range properly). The Fairness Index value becomes 1 for the whole topology if each node belonging to the network has a probability to access the channel equal to 1 . Here, we want to clearly state that the Fairness Index value varies with time. Since the rate changes in CARA and in REFOT, depending on the channel condition, a node may have different transmission rates at different times. Similarly, if the nodes that are present in the network are mobile, the probability to access the channel is dynamically estimated in our scheme, while this is not the case for CARA and Classical RTS/CTS approaches. Therefore, the Fairness Index value may vary with time and other parameters, such as: the channel quality, the number of competitor nodes, etc. Thus, we take an average value of this function over the simulation time to have the Fairness Index of a given topology in the whole simulation.

\section{Simulation SETUP AND PERFORMANCE ANALYSIS}

In this section, we discuss the simulation's parameters and compare REFOT, CARA and Classical RTS/CTS approaches. We implemented these schemes in NS-2 [7] in order to simulate them with different simulation's parameters. Simulation is done for static complex topology illustrated in figure 1 and various topologies with random way point mobility model for 10 and 20 nodes with several transmission ranges. Furthermore, we compared the impact of the different approaches with the UDP connections on the $F I$ and on the throughput.

\section{A. A network case study}

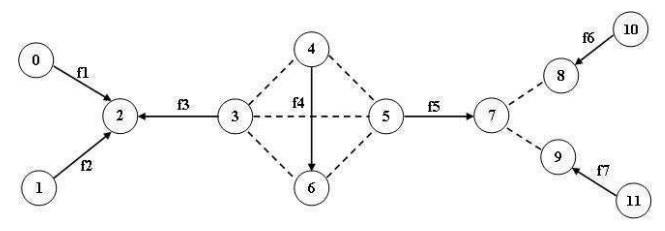

Fig. 1. A complex network topology

We consider a network with 12 static nodes as shown in figure 1 . We have flows $f_{1}, f_{2}$ and $f_{3}$ which are in the same contention range and thus share one common channel. Similarly, $f_{3}, f_{4}$ and $f_{5}$ share one common channel while flows $f_{5}, f_{6}$ and $f_{7}$ share another common channel. All three channels are different. Since in our scheme fairness is measured on how a node respects other contenders to have access to the common resources, according to the Fairness Index $(F I)$ equation 4, we have plotted the result of our simulation, $F I$ versus simulation time. According to the result in figure 2, we can easily deduce that REFOT is much better to have a relative fairness among the senders. In the graph, we see that till $20 \mathrm{sec}$ the fairness index values are almost near to those of CARA and Classical RTS/CTS schemes but later, it raises and becomes significantly different. The probability to access the channel plays a key role in the evaluation of the Fairness Index ; its value is estimated by overhearing. Thus, a few seconds are required to correctly gather the information about the neighborhood so that a sender can estimate the

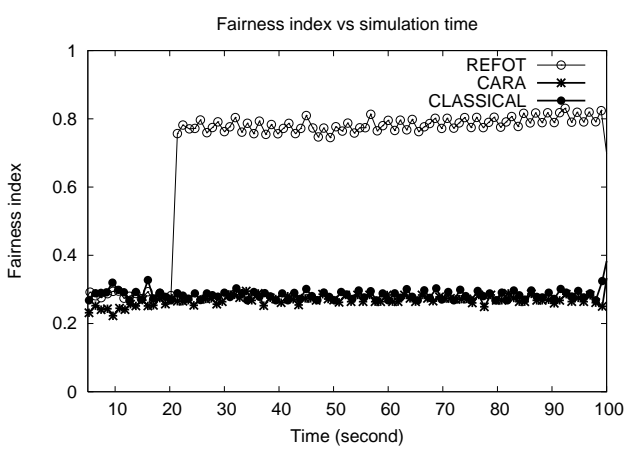

Fig. 2. Fairness index versus simulation time with UDP flows

probability to access the channel in a more accurate way. After 20 seconds, we see a very significant improvement in the fairness index result. With figure 3 , one can easily deduce that

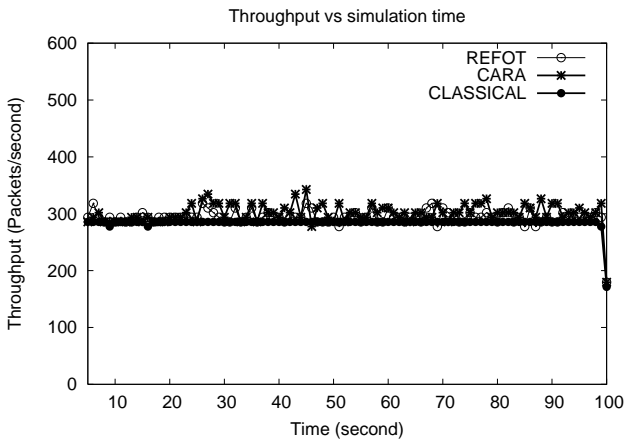

Fig. 3. Throughput versus simulation time with UDP flows

our scheme does not degrade the throughput in comparison with CARA and Classical RTS/CTS schemes. It is nearly the same throughout according to the simulation time.

\section{B. Impact of network density on fairness and throughput}

Let use now discuss the various topologies with random way point mobility model with varying the transmission ranges. We took the max speed as $5 \mathrm{~m} / \mathrm{sec}$, under the area of $800 m x 800 m$ with TwoRayGround as propagation model. We varied the transmission range from $25 \mathrm{~m}$ to $250 \mathrm{~m}$ for a topology consisting of 10 to 20 nodes.

1) Case of 10 nodes with different transmission ranges: Figure 4 shows that REFOT ensures a much better fairness in comparison with CARA and Classical RTS/CTS approaches. We note that the $F I$ value is approximately $40 \%$ greater than in CARA and Classical RTS/CTS schemes. Since CARA and Classical RTS/CTS approaches do not take into account the fairness with adapting rate among other contenders of the channel, both have a lower value of $F I$. It is obvious that, as the transmission range increases, the number of contenders in the channel increases, and hence, the probability to access the channel may decrease. Therefore, the FI may decrease while the transmission range increases. In figure 5, we notice that our approach has nearly the same throughput in comparison 


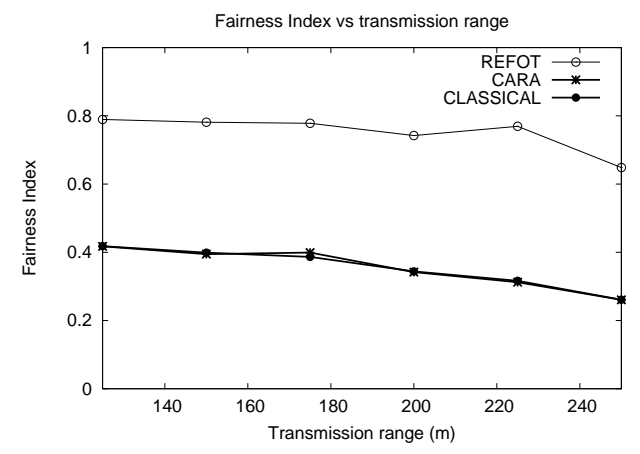

Fig. 4. Fairness index versus transmission range (10 nodes with UDP flows)

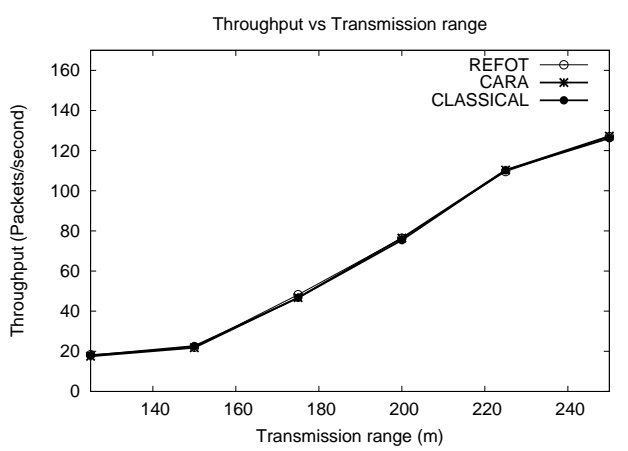

Fig. 5. Throughput versus transmission range (10 nodes with UDP flows)

with CARA and classical RTS/CTS approaches. Our scheme gives a much better fairness among the contenders of the channel without negatively affect the throughput. We already pointed out that creating an absolute fairness and optimizing the throughput has a tradeoff, and our scheme gives a balance between these two aspects.

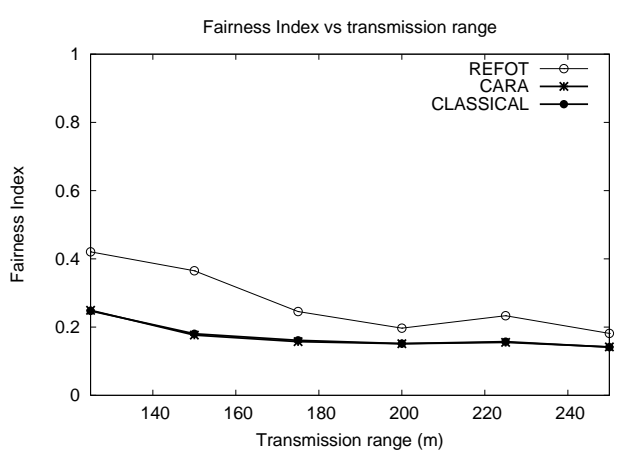

Fig. 6. Fairness index versus transmission range (20 nodes with UDP flows)

2) Case of 20 nodes with different transmission range: We set the total number of nodes to 20 and we plot the obtained results in figure 6 . We note that the $F I$ decreases by $50 \%$ in comparison with the first case (10 nodes). However, it is much better in comparison with CARA and Classical RTS/CTS approaches. We remark that the $F I$ value is always more than $30 \%$ increment in value compared to the other schemes. In order to illustrate the impact on the throughput, we

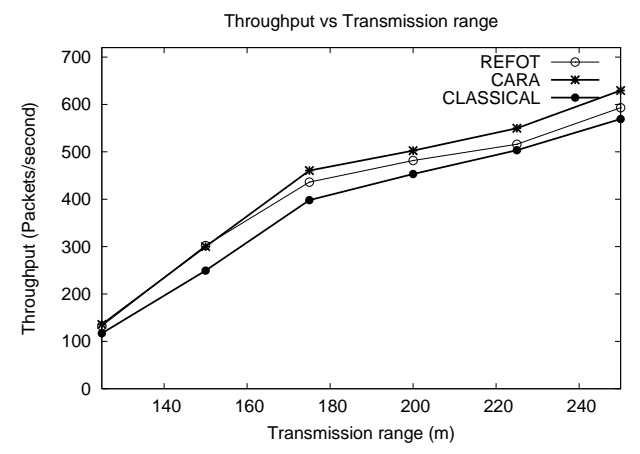

Fig. 7. Throughput versus transmission range (20 nodes with UDP flows)

plot figure 7. We remark that the throughput in our approach is a little less than CARA scheme but better than classical RTS/CTS schemes. When the transmission range increases, the $F I$ decreases but the throughput increases ; we can have more ways to reach the destination, because in ad-hoc networks, the communication is based on multi-hops connection. All these results clearly prove that our scheme REFOT ensures a better tradeoff between fairness and throughput in different transmission ranges and with different number of nodes.

\section{CONCLUSION}

In this paper, we have proposed an analytical model to adapt the transmission rate and to create a relative fairness among the competitors of the common channels without decreasing the throughput significantly in comparison with other approaches. The estimation of probability to access the channel that is dynamically calculated plays a key role in our approach. We have defined a fairness index which is a metric to estimate the fairness in the topology, which depends on the probability to access the channel. We have shown in various simulations that our scheme outperforms others in any complex scenario to create fairness without decreasing the throughput significantly. Hence our approach provides a balance between having a relative fairness in adapting the rate and having a good throughput. A future work is to study and analyse our scheme with different assumptions and conditions.

\section{REFERENCES}

[1] Ad Kamerman and Leo Monteban, "WaveLAN-II: a high-performance Wireless LAN for the Unlicensed Band," Bell Labs Technical Journal, vol.2, no.3, pp.118-133, Aug. 1997.

[2] P. Chevillat, J. Jelitto, A. Noll Barreto, and H. L. Truong, A Dynamic Link Adaptation Algorithm for IEEE 802.11a Wireless LANs, in Proc. IEEE ICC'03, Anchorage, AK, May 2003, pp. 1141-1145.

[3] Javier del Prado Pavon and Sunghyun Choi, Link Adaptation Strategy for IEEE 802.11 WLAN via Received Signal Strength Measurement, in Proc. IEEE ICC'03, Anchorage, AK, May 2003.

[4] J. Kim, S. Kim, S. Choi, and D. Qiao, CARA: Collision-Aware Rate Adaptation for IEEE 802.11 WLANS, in Proc. 25th IEEE International Conference on Computer Communications (INFOCOM 2006), 2006.

[5] Gavin Holland, Nitin Vaidya, and Paramvir Bahl, A Rate-Adaptive MAC Protocol for Multi-Hop Wireless Networks, in Proc. ACM Mo-biCom'01, Rome, Italy, Jul. 2001.

[6] IEEE 802.11, IEEE802.11 wireless LAN media access control (MAC) and physical layer (PHY) specifications, 1999.

[7] UC Berkeley and USC ISI, The network simulator ns-2, Part of the VINT project. Available from http://www.isi.edu/nsnam/ns, 1998. 